\title{
IncRNA MEG8 Upregulates miR-770-5p Through Methylation and Promotes Cell Apoptosis in Diabetic Nephropathy
}

This article was published in the following Dove Press journal: Diabetes, Metabolic Syndrome and Obesity: Targets and Therapy

\author{
Jinmei Zhang' \\ Liwen Song ${ }^{2}$ \\ Yanjuan $\mathrm{Ma}^{3}$ \\ Yan Yin' \\ Xinqi Liu' \\ Xinyu Luo' \\ Jiali Sun' \\ Liqin Wang' \\ 'Department of Endocrinology, Weifang \\ Hospital of Traditional Chinese Medicine, \\ Weifang, Shandong Province 261000, \\ People's Republic of China; ${ }^{2}$ Department \\ of Endocrinology, Weifang People's \\ Hospital, Weifang, Shandong Province \\ 261000, People's Republic of China; \\ ${ }^{3}$ Department of Endocrinology, Sunshine \\ Fusion Hospital, Sunshine, Shandong \\ Province 26106I, People's Republic of \\ China
}

Background: It has been reported that lncRNA MEG8 can be induced by glucose in mice model of kidney injury, indicating its role in diabetic nephropathy (DN). This study was carried out to explore the role of MEG8 in DN.

Materials and Methods: The expression of MEG8 and miR-770-5p in plasma samples from DN patients $(n=66)$, diabetic patients (DM patients with no complications, $n=66)$ and healthy controls $(n=66)$ was detected by RT-qPCR. The interaction between MEG8 and miR-770-5p in podocyte cells was evaluated by transient transfections. Cell apoptosis under high-glucose treatment was detected by cell apoptosis assay.

Results: MEG8 and miR-770-5p were upregulated in plasma of DM patients and were further upregulated in DN patients. MEG8 was positively correlated with miR-770-5p. In podocyte cells, high-glucose treatment resulted in increased expression levels of MEG8 and miR-770-5p. In podocyte cells, overexpression of MEG8 resulted in upregulated expression of miR-770-5p and decreased methylation of the miR-770-5p gene. Cell apoptosis analysis showed that overexpression of MEG8 and miR-770-5p resulted in increased cell apoptotic rate under glucose treatment. In addition, combined overexpression of MEG8 and miR-770$5 \mathrm{p}$ showed stronger effects.

Conclusion: MEG8 may upregulate miR-770-5p through methylation to promote DN by promoting cell apoptosis.

Keywords: diabetic nephropathy, MEG8, miR-770-5p, apoptosis

\section{Introduction}

Diabetes mellitus (DM) is one of the major public health concerns with increasing incidence rate in recent years. ${ }^{1}$ International Diabetes Federation (IDF) estimated that there were about 415 million people with diabetes in 2015 , and this number will be increased to 642 million by $2040 .^{2} \mathrm{DM}$ is a chronic disease that causes multiple complications that affect almost all important organs in the human body. ${ }^{3}$ In a considerable portion of DM patients, hyperglycemia causes damages to the kidney, which filters blood, leading to the development of diabetic nephropathy (DN). ${ }^{4}$ Lesions caused by DN are usually irreversible. ${ }^{5}$ Without timely treatment, DN will eventually develop into end-stage renal failure, which is related to the high mortality rate. ${ }^{6,7}$

Novel therapeutic approaches are needed to improve the treatment of DN. ${ }^{8}$ With the increased understanding of the molecular mechanisms of $\mathrm{DN}$, various molecular players have been characterized in this disease. ${ }^{8}$ In effect, some molecular players, such as Sirt1 and IL-17a, have been identified as potential targets for the treatment of
Correspondence: Liwen Song Department of Endocrinology, Weifang People's Hospital, Weifang City, Shandong Province 261000, People's Republic of China

Tel +86536-963360

Email zjmmjmdr@I26.com 
DN. ${ }^{9,10}$ Although non-coding RNAs (ncRNAs) encode no protein products, they regulate gene expression and participate in the development and progression of human diseases. $^{11,12}$ Therefore, ncRNAs, such as lncRNAs, are also potential targets for the treatment of DN. It has been reported that lncRNA MEG8 and miR-770-5p both promote $\mathrm{DN},{ }^{13,14}$ indicating the potential interactions between them. This study was carried out to investigate the interaction between MEG8 and miR-770-5p in DN.

\section{Materials and Methods}

\section{Patients and Healthy Controls}

This study enrolled $66 \mathrm{DN}$ patients (36 males and 30 females; 44 to 69 years old; mean age $56.7 \pm 5.2$ years old), $66 \mathrm{DM}$ patients (36 males and 30 females; 44 to 69 years old; mean age $56.6 \pm 5.4$ years old) and 66 healthy controls (36 males and 30 females; 44 to 69 years old; mean age $56.8 \pm 5.3$ years old) at Weifang People's Hospital between June 2017 and June 2019. The Ethics Committee of Weifang People's Hospital approved this study before the enrollment of patients and controls. All the experimental procedures were conducted in accordance with the Declaration of Helsinki. All DN and DM patients were diagnosed for the first time and no initiated therapy or other clinical disorders were observed. All participants received systemic physiological exams. The healthy controls showed normal functions for all critical organs. DN and DM patients were excluded from other severe clinical disorders. All participants provided written

Table I Basic Information of Three Groups of Participants

\begin{tabular}{|l|l|l|l|}
\hline Indexes & DN & DM & Control \\
\hline Cases & 66 & 66 & 66 \\
Males & 36 & 36 & 36 \\
Females & 30 & 30 & 30 \\
Duration of & $11.8 \pm 3.3$ & $6.9 \pm 3.5$ & $\mathrm{NA}$ \\
diabetes (years) & & & \\
BMI (kg/m ${ }^{2}$ ) & $24.14 \pm 2.06$ & $23.76 \pm 2.22$ & $22.91 \pm 1.39$ \\
Systolic blood & $134.38 \pm$ & $128.34 \pm 4.98$ & $127.41 \pm 4.36$ \\
pressure (mmHg) & 7.11 & & \\
Diastolic blood & $81.33 \pm 4.54$ & $77.36 \pm 7.36$ & $77.14 \pm 4.99$ \\
pressure (mmHg) & & & \\
Fasting blood sugar & $153.37 \pm$ & $123.54 \pm$ & $63.11 \pm 33.12$ \\
(mg/dL) & 34.24 & 51.78 & \\
HbAlc (mmol/ & $83.77 \pm 9.78$ & $71.71 \pm 8.41$ & $35.71 \pm 4.41$ \\
mol) & & & \\
Serum urea & $30.10 \pm 6.56$ & $22.55 \pm 6.84$ & $18.324 \pm 4.12$ \\
Serum Creatinine & $1.18 \pm 0.29$ & $0.92 \pm 0.20$ & $0.83 \pm 0.11$ \\
\hline
\end{tabular}

form of the informed consent. See Table 1 for the basic information of three groups of participants.

\section{Blood Extraction and Preparation of Plasma Samples}

All patients and controls were fasted for more than $12 \mathrm{~h}$, followed by extraction of blood $(5 \mathrm{~mL})$ from the elbow vein. To prepare plasma samples, blood samples were centrifuged in EDTA tubes at $1200 \mathrm{~g}$ at room temperature for $18 \mathrm{~min}$. The supernatant, which was plasma, was collected and immediately subjected to RNA isolations.

\section{Podocytes}

Human podocyte cell line CIHP-1 (Ximbio) was used. Cells were cultivated in a medium composed of FBS (10\%) and RPMI-1640 (90\%) supplemented with InsulinTransferrin-Selenium and Pen/Strep. Cells were cultured at $37{ }^{\circ} \mathrm{C}$ with $5 \% \mathrm{CO}_{2}$. Cells were collected from passage 3 to 6 to be used in the following experiments. In cases of high-glucose treatment, CIHP-1 cells were cultivated in fresh medium containing 10, 20 and $40 \mathrm{mM}$ D-glucose (Sigma-Aldrich) for $48 \mathrm{~h}$ before use.

\section{Cell Transfection}

MEG8 expressing vector was constructed by Sangon (Shanghai, China) with pcDNA3.1 vector as backbone. To overexpress miR-770-5p, mimic of miR-770-5p and negative control (NC) miRNA were synthesized by Sangon. Vector (1 $\mu \mathrm{g})$ and/or miRNA (40 $\mathrm{nM})$ was transfected into CIHP-1 cells using Lipofectamine 2000 (Invitrogen) following the manufacturer's instructions. Untransfected cells were used as Control (C) cells. NC miRNA- or empty vector-transfected cells were NC cells. Cells collected at $48 \mathrm{~h}$ post-transfection were used in subsequent experiments.

\section{RNA Preparations}

Total RNAs were isolated from CIHP-1 cells and plasma samples using Ribozol (Invitrogen). To harvest miRNAs, RNA samples were precipitated and washed using ice-cold $85 \%$ ethanol. RNA samples were digested with DNase I at $37{ }^{\circ} \mathrm{C}$ for $1 \mathrm{~h}$ to digest genomic DNAs. RNA integrity was checked using a Urea-PAGE gel $(6 \%)$.

\section{RT-qPCR}

A reverse transcription system (A5003, Promega Corporation) was used to reverse transcribe RNA samples into cDNA samples. The qPCRs reactions were prepared using QuantiTect SYBR Green PCR Kit (QIAGEN). Human 
18S rRNA was used as the internal control to determine the expression levels of MEG8. The expression levels of mature miR-770-5p were determined through the following steps: 1) addition of poly (A) to mature miRNAs; 2) reverse transcriptions; 3) qPCRs. All of the three steps were performed using All-in-One ${ }^{\mathrm{TM}}$ miRNA qRT-PCR Detection Kit (Genecopoeia). U6 was used as the internal control of miR770-5p. Primer sequences were as following: 5'CTCTGTGAATCAGGAGAGAAGAGA-3' (forward) and 5'-TTCACCTTGGGGAAATGACCA-3' (reverse) for MEG8; 5'-GTAACCCGTTGAACCCCATT-3' (forward) and 5'-CCATCCAATCGGTAGTAGCG-3' (reverse) for MEG8 for 18S rRNA. Forward primer of miR-770-5p was 5'-TCCAGTACCACGTGTCAGGGCCA-3'. U6 forward primer and universal reverse primers were from the kit. Three replicates were included in each experiment. Ct values were normalized using $2^{-\Delta \Delta C T}$ method.

\section{Cell Apoptosis Assay}

CIHP-1 cells were collected from each transfection group at $48 \mathrm{~h}$ post-transfection. Cells were cultivated in medium containing $40 \mathrm{nM}$ D-glucose for $48 \mathrm{~h}$, followed by washing with ice-cold PBS. After that, Annexin V-FITC (Dojindo, Japan) and propidium iodide (PI) were used to stain the cells for $20 \mathrm{~min}$ in dark. Finally, flow cytometry was used to analyze apoptotic (early apoptosis) cells.

\section{Methylation-Specific PCR (MSP)}

CIHP-1 cells were subjected to genomic DNA isolation using Monarch ${ }^{\circledR}$ Genomic DNA Purification Kit (NEB). EZ DNA Methylation-Gold ${ }^{\mathrm{TM}}$ kit (ZYMO) was used to convert DNA samples. Taq 2X Master Mix (M0270, NEB) was used to perform all PCRs to analyze the methylation of miR-770-5p. MSP primers were designed to clone methylated miR-770-5p gene and non-MSP primers were used to clone un-methylated miR-770-5p gene. MSP primers were: 5'-AGGAGCCACCTTCCGAGC-3' (forward) and 5'-AAGAGCAGACCCCTGAGG-3' (reverse). NonMSP primers were: 5'-AGGAGTTATTTTTCGAGT-3' (forward) and 5'-AAAAACAAACCCCTAAAA' (reverse).

\section{Statistical Analysis}

Comparisons among multiple groups were explored by ANOVA Tukey's test. Data of triplicates were expressed as mean or mean \pm standard deviation (SD) values. Linear regression analysis was performed for correlation analysis. Differences were considered as statistically significant when $p<0.05$.

\section{Results}

The Expression of MEG8 and miR-770-5p Were Both Upregulated in DN

The expression of MEG8 and miR-770-5p in plasma from DN patients $(n=66)$, DM patients $(n=66)$ and controls $(n$ =66) were detected by RT-qPCR. RT-qPCR results showed that, compared to the Control group, DN and DM groups exhibited significantly higher expression levels of MEG8 (Figure 1A, $p<0.01$ ) and miR-770-5p (Figure $1 \mathrm{~B}, p<0.01)$. Moreover, DN group also exhibited significantly higher expression levels of MEG8 and miR-770-5p in comparison to that in DM group $(p<0.05)$.

\section{The Expression of MEG8 and miR-770-5p Were Positively Correlated Only Across Plasma Samples from DN Patients}

Correlations between the expression levels of MEG8 and miR-770-5p across plasma samples from DN patients
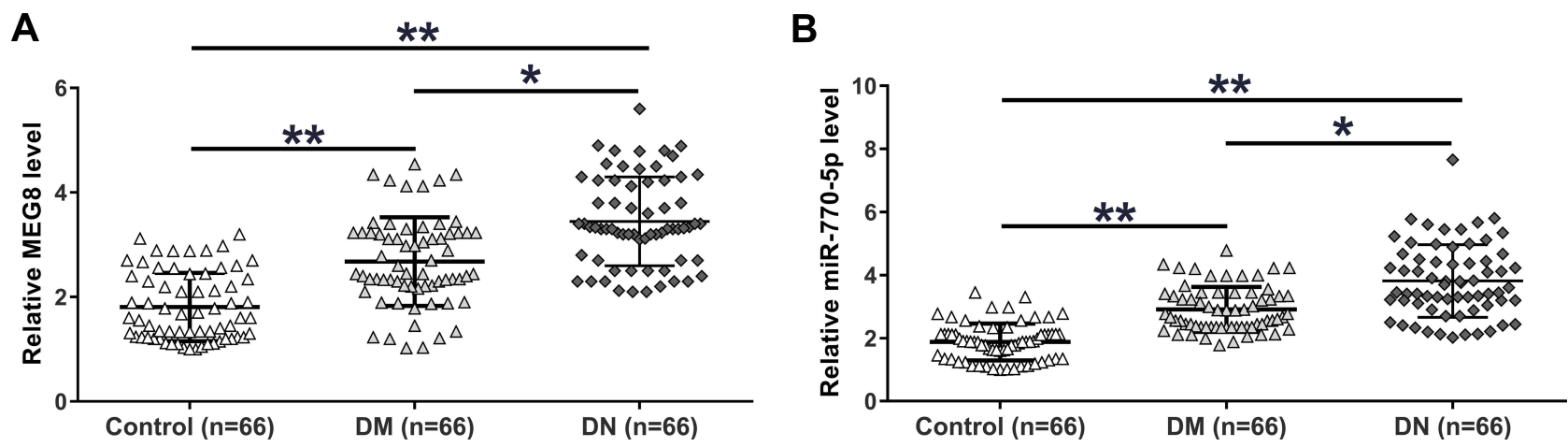

Figure I The expression of MEG8 and miR-770-5p were both upregulated in DN. Expression of MEG8 $(\mathbf{A})$ and miR-770-5p $(\mathbf{B})$ in plasma from DN patients $(\mathrm{n}=66)$, DM patients $(n=66)$ and controls $(n=66)$ were analyzed by RT-qPCR. Mean values of 3 replicates were presented. $* p<0.05 ; * *<<0.01$. 
(Figure 2A), DM patients (Figure 2B) and controls (Figure 2C) were analyzed by linear regression. It was observed that the expression of MEG8 and miR-770-5p were positively correlated only across plasma samples from $\mathrm{DN}$ patients, but not DM patients and controls.

\section{MEG8 Upregulated the Expression of miR-770-5p in CIHP-I Cells by Reducing the Methylation of miR-770-5p}

To explore the interaction between MEG8 and miR-770$5 \mathrm{p}$ in DN, CIHP-1 cells were treated with $40 \mathrm{nM}$ D-glucose for $48 \mathrm{~h}$ to mimic DN, followed by transfections of MEG8 expression vector or miR-770-5p mimic. Transfections were confirmed by RT-qPCR (Figure 3A, $p<0.05)$. It was observed that cells with MEG8 expression vector transfection also exhibited upregulation of
miR-770-5p (Figure 3B, $p<0.05$ ), while cells with overexpression of miR-770-5p showed no significantly altered expression of MEG8 (Figure 3C, $p>0.05$ ). MSP was performed to assess the effects of overexpression of MEG8 on the methylation of miR-770-5p. Compared to cells transfected with empty vector, cells transfected with MEG8 expression vector exhibited obviously reduced methylation of miR-770-5p (Figure 3D).

\section{Glucose-Inducible MEG8 and miR-770-5p Promoted Glucose-Mediated Apoptosis of CIHP-I Cells}

CIHP-1 cells were cultivated in medium containing 10, 20 and $40 \mathrm{mM}$ D-glucose for $48 \mathrm{~h}$, followed by determination of the expression of MEG8 and miR-770-5p by RT-qPCR. It was observed that D-glucose treatment resulted in

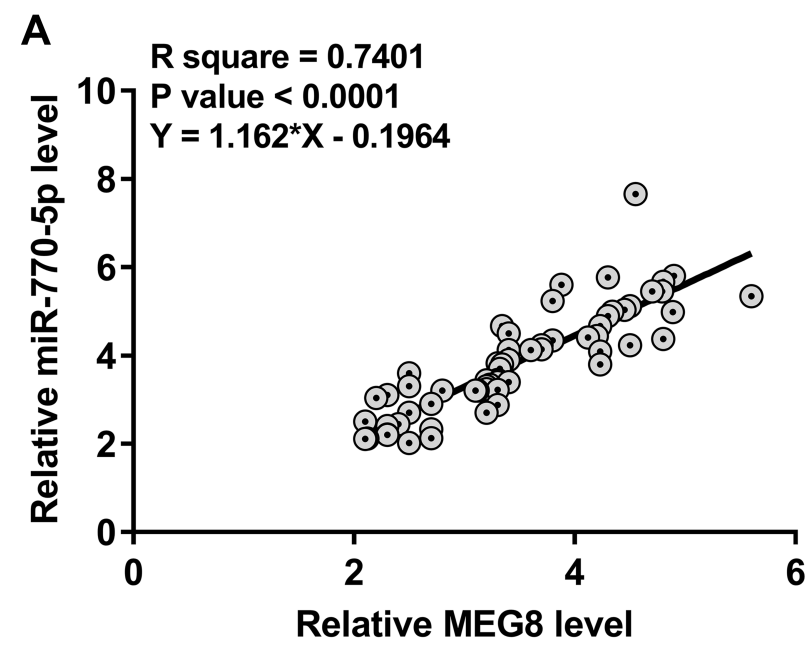

B
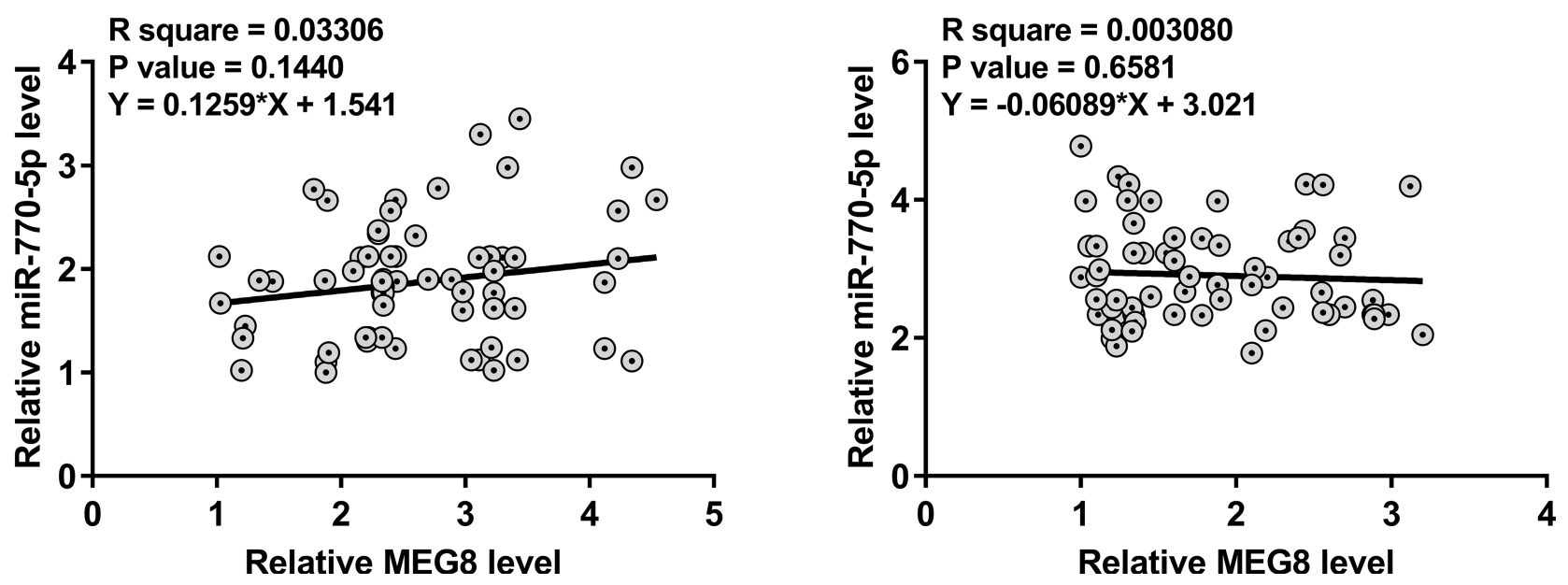

Figure 2 MEG8 and miR-770-5p were positively correlated only across plasma samples from DN patients. Correlations between the expression levels of MEG8 and miR770-5p across plasma samples from DN patients (A), DM patients (B) and controls (C) were analyzed by linear regression. 

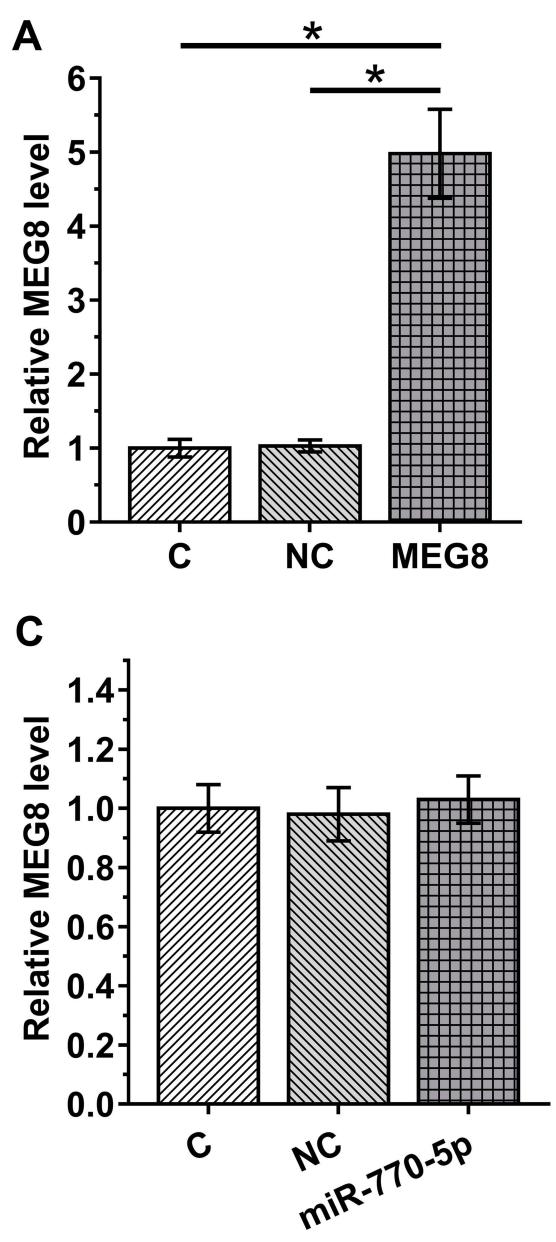

B
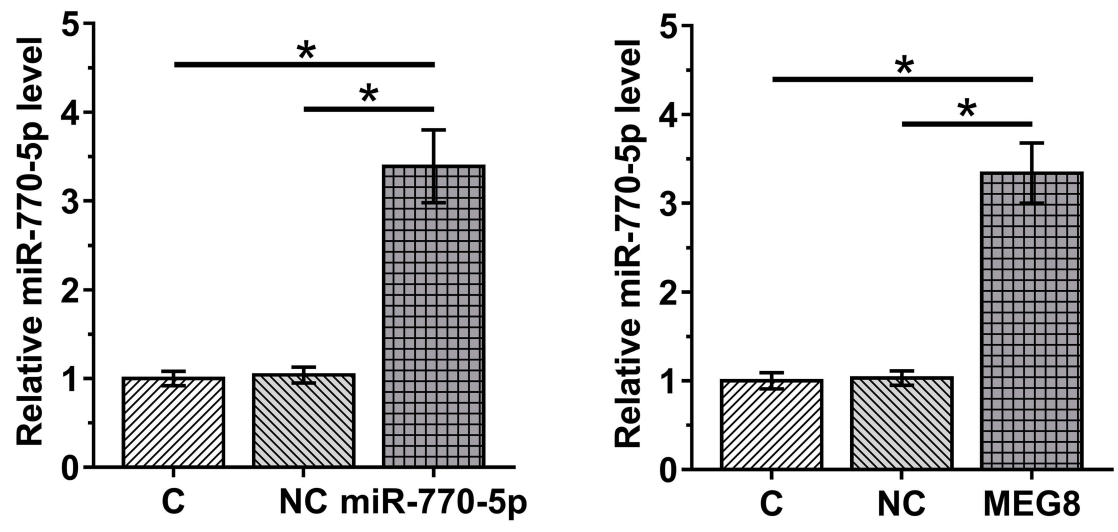

D

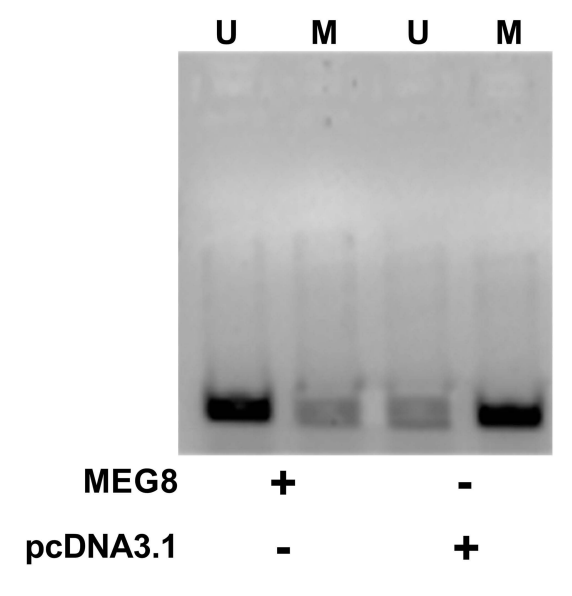

Figure 3 MEG8 upregulated the expression of miR-770-5p in CIHP-I cells by reducing the methylation of miR-770-5p. To explore the interaction between MEG8 and miR770-5p in DN, ClHP-I cells were treated with $40 \mathrm{nM}$ D-glucose for $48 \mathrm{~h}$ to mimic DN, followed by transfections of MEG8 expression vector or miR-770-5p mimic. Transfections were confirmed by RT-qPCR (A). The effects of MEG8 expression vector transfection on the expression of miR-770-5p (B), and the effects of overexpression of miR-770-5p on MEG8 (C) were also analyzed by RT-qPCR. MSP was performed to analyze the effects of overexpression of MEG8 on the methylation of miR-770-5p. In MSP, MSP primers were designed to clone methylated miR-770-5p gene and non-MSP primers were used to clone un-methylated miR-770-5p gene in cells transfected with MEG8 expression vector or empty pcDNA3.I vector. PCR products were subjected to $1 \%$ agarose gel electrophoresis, followed by ethidium bromide staining (D). Mean \pm $\mathrm{SD}$ values of 3 replicates were presented. $U$, un-methylation; $M$, methylation; ${ }^{*} p<0.05$.

increased expression levels of MEG8 (Figure 4A) and miR-770-5p (Figure 4B) in a dose-dependent manner $(p<0.05)$. Cell apoptosis assay was performed to explore the role of MEG8 and miR-770-5p in regulating the apoptosis of CIHP-1 cells. It was observed that overexpression of MEG8 and miR-770-5p resulted in increased cell apoptotic rate under glucose treatment. In addition, combined overexpression of MEG8 and miR-770-5p showed stronger effects (Figure 4C).

\section{Discussion}

This study investigated the interaction between MEG8 and miR-770-5p in DN. We found that MEG8 and miR-770-5p were both upregulated in DN. Moreover, MEG8 and miR$770-5 p$ were both inducible by high glucose and the role of
MEG8 in promoting the apoptosis of human podocytes is likely associated with miR-770-5p.

A recent study reported that the expression of MEG8 could be induced by glucose in mice model of kidney injury, ${ }^{14}$ indicating its role in DN. In this study, we showed that MEG8 was upregulated in DN and its expression in human podocytes was upregulated by treatment with high glucose. In addition, overexpression of MEG8 resulted in increased apoptotic rate of podocytes. In the kidneys of DN patients, apoptosis of podocytes is accelerated, leading to kidney injury. ${ }^{15,16}$ Therefore, glucose-inducible MEG8 may promote the development of DN by promoting the apoptosis of podocytes. Interestingly, MEG8 was also upregulated in DM patients without obvious complications. Therefore, MEG8 may also participate in other types of diabetic 
A

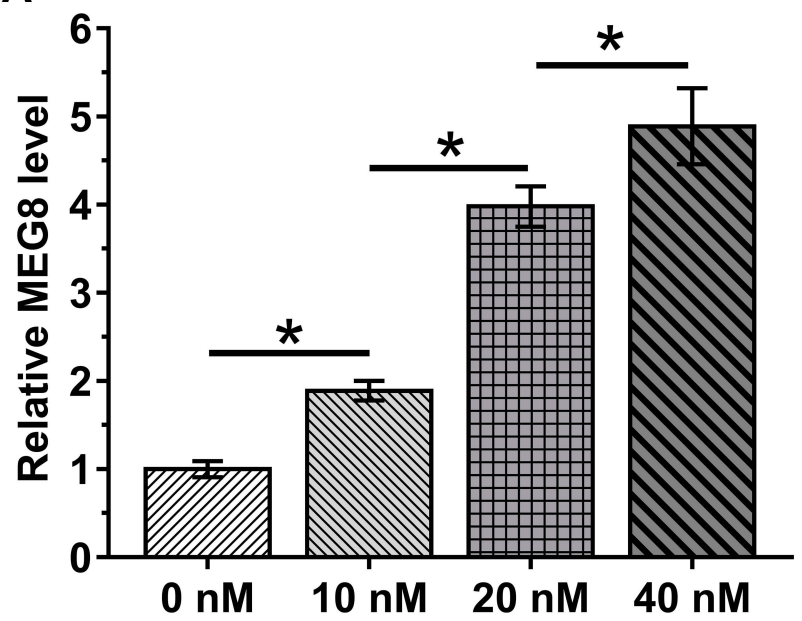

B

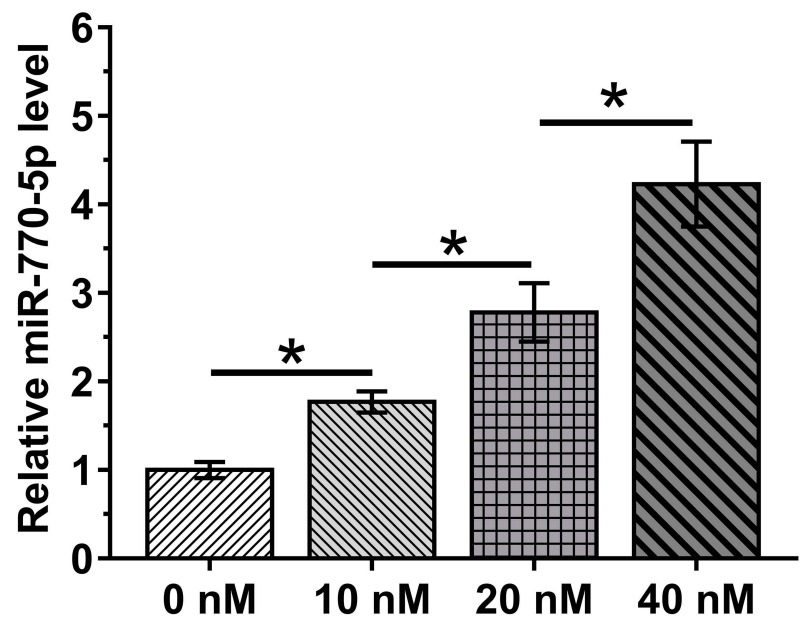

C

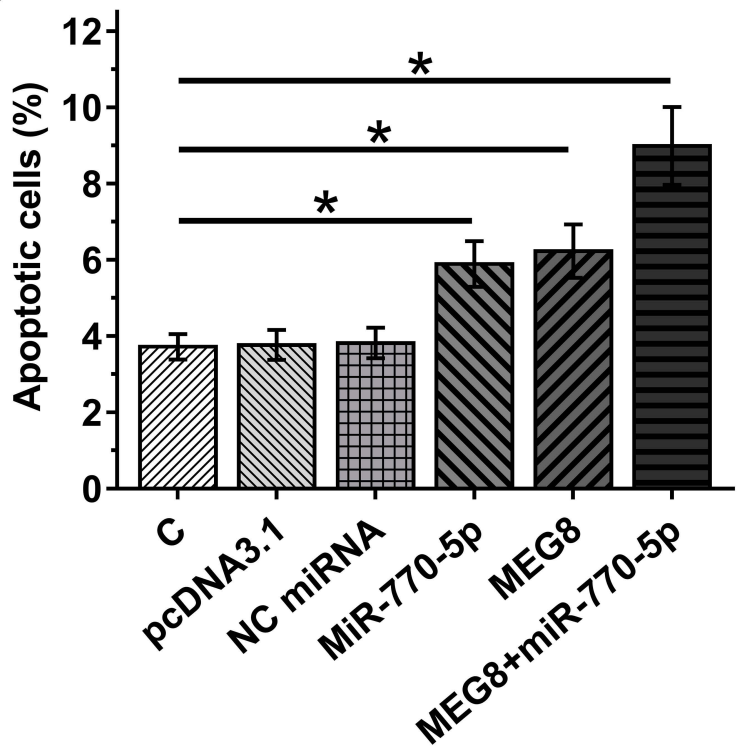

Figure 4 Glucose-inducible MEG8 and miR-770-5p promoted glucose-mediated apoptosis of ClHP-I cells. CIHP-I cells were cultivated in medium containing I0, 20 and 40 $\mathrm{mM}$ D-glucose for $48 \mathrm{~h}$, followed by determining the expression of MEG8 (A) and miR-770-5p (B) by RT-qPCR. Cell apoptosis assay was performed to explore the role of MEG8 and miR-770-5p in regulate the apoptosis of CIHP-I cells $(\mathbf{C})$. Mean \pm SD values of 3 replicates were presented. ${ }^{*} p<0.05$.

complication. Future studies are needed to further explore the role of MEG8 in diabetic complications.

MiR-770-5p was reported to target TP53 regulated inhibitor of apoptosis 1 to induce the apoptosis of podocytes, thereby promoting the development of DN. ${ }^{13}$ Consistent with previous studies, this study reported the upregulation of miR-770-5p in DN and its enhancing effects on glucoseinduced apoptosis of podocytes. Interestingly, our study showed that miR-770-5p could be upregulated in podocytes by MEG8, and the upregulation of miR-770-5p by MEG8 is at least partially achieved through the role of MEG8 in reducing the methylation of miR-770-5p gene. However, the interaction between miR-770-5p and MEG8 is likely DN-specific. This is because miR-770-5p and MEG8 were only closely correlated across DN patients but not DM patients and healthy controls. Interestingly, cell apoptosis analysis showed that MEG8 overexpression has slightly stronger effects on cell apoptosis in comparison to overexpression of miR-770-5p. It may be caused by the different overexpression rates of these two factors. Another possibility is that MEG8 may also interact with other apoptotic factors. Further studies are needed.

It has been reported that MEG8 is overlapped with miR-379 microRNA cluster and 14q32 miRNA cluster. MiRNAs in miR-379 microRNA cluster are upregulated in kidney and glomeruli from diabetic mice and mesangial cells treated with HG and TGF-b. In addition, it is known that miR-379 can target EDEM3 and ATF3 to regulate ER 
stress. ${ }^{17} 14 \mathrm{q} 32$ miRNA cluster plays critical roles in kidney fibrosis. ${ }^{18}$ Our future studies will explore the interactions between MEG8, miR-379 and 14q32 miRNA cluster in $\mathrm{DN}$.

\section{Conclusions}

In conclusion, miR-770-5p and MEG8 are both upregulated in DN and they may induce glucose-induced apoptosis of podocytes to participate in DN. In addition, MEG8 may upregulate miR-770-5p by reducing the methylation of miR-770-5p gene.

\section{Disclosure}

The authors report no conflicts of interest in this work.

\section{References}

1. Cukic I, Weiss A. Personality and diabetes mellitus incidence in a national sample. $J$ Psychosom Res. 2014;77(3):163-168. doi:10.1016/j.jpsychores.2014.07.004

2. Zimmet P, Alberti KG, Magliano DJ, Bennett PH. Diabetes mellitus statistics on prevalence and mortality: facts and fallacies. Nat Rev Endocrinol. 2016;12(10):616-622. doi:10.1038/nrendo.2016.105

3. Forbes JM, Cooper ME. Mechanisms of diabetic complications. Physiol Rev. 2013;93(1):137-188. doi:10.1152/physrev.00045.2011

4. Duran-Salgado MB, Rubio-Guerra AF. Diabetic nephropathy and inflammation. World J Diabetes. 2014;5(3):393-398. doi:10.4239/ wjd.v5.i3.393

5. Lim A. Diabetic nephropathy - complications and treatment. Int J Nephrol Renovasc Dis. 2014;7:361-381. doi:10.2147/IJNRD. S40172

6. Kelly KJ, Dominguez JH. Rapid progression of diabetic nephropathy is linked to inflammation and episodes of acute renal failure. $A m$ J Nephrol. 2010;32(5):469-475. doi:10.1159/000320749

7. Cao Z, Cooper ME. Pathogenesis of diabetic nephropathy. J Diabetes Investig. 2011;2(4):243-247. doi:10.1111/j.2040-1124.2011.00131.x
8. Sharma D, Bhattacharya P, Kalia K, Tiwari V. Diabetic nephropathy: new insights into established therapeutic paradigms and novel molecular targets. Diabetes Res Clin Pract. 2017;128:91-108. doi:10.10 16/j.diabres.2017.04.010

9. Kume S, Kitada M, Kanasaki K, Maegawa H, Koya D. Anti-aging molecule, Sirt1: a novel therapeutic target for diabetic nephropathy. Arch Pharm Res. 2013;36(2):230-236. doi:10.1007/s12272-0130019-4

10. Lavoz C, Rayego-Mateos S, Orejudo del Río M, et al. Could IL-17A Be a Novel Therapeutic Target in Diabetic Nephropathy? J Clin Med. 2020;9:272.

11. Shi X, Sun M, Liu H, Yao Y, Song Y. Long non-coding RNAs: a new frontier in the study of human diseases. Cancer Lett. 2013;339 (2):159-166. doi:10.1016/j.canlet.2013.06.013

12. Alvarez ML, Distefano JK. The role of non-coding RNAs in diabetic nephropathy: potential applications as biomarkers for disease development and progression. Diabetes Res Clin Pract. 2013;99(1):1-11. doi:10.1016/j.diabres.2012.10.010

13. Zhang SZ, Qiu XJ, Dong SS, et al. MicroRNA-770-5p is involved in the development of diabetic nephropathy through regulating podocyte apoptosis by targeting TP53 regulated inhibitor of apoptosis 1. Eur Rev Med Pharmacol Sci. 2019;23(3):1248-1256. doi:10.26355/ eurrev $201902 \quad 17018$

14. Tsotakos N, Caldwell EA, Cerny G, Hartman HL, Saade N. Glucoseinduced dysregulation of lncRNAs in a model of chronic kidney disease. FASEB J. 2019;33(1_supplement):778.719.

15. Mundel P, Kriz W. Structure and function of podocytes: an update. Anat Embryol (Berl). 1995;192(5):385-397. doi:10.1007/BF002 40371

16. Li JJ, Kwak SJ, Jung DS, et al. Podocyte biology in diabetic nephropathy. Kidney Int Suppl. 2007;72(106):S36-42. doi:10.1038/ sj.ki.5002384

17. Kato $M$, Wang $M$, Chen $Z$, et al. An endoplasmic reticulum stress-regulated lncRNA hosting a microRNA megacluster induces early features of diabetic nephropathy. Nat Commun. 2016;7:12864. doi:10.1038/ncomms 12864

18. Bijkerk R, Au YW, Stam W, et al. Long non-coding RNAs rian and miat mediate myofibroblast formation in kidney fibrosis. Front Pharmacol. 2019;10:215. doi:10.3389/fphar.2019.00215

Diabetes, Metabolic Syndrome and Obesity: Targets and Therapy

Dovepress

\section{Publish your work in this journal}

Diabetes, Metabolic Syndrome and Obesity: Targets and Therapy is an international, peer-reviewed open-access journal committed to the rapid publication of the latest laboratory and clinical findings in the fields of diabetes, metabolic syndrome and obesity research. Original research, review, case reports, hypothesis formation, expert opinion and commentaries are all considered for publication. The manuscript management system is completely online and includes a very quick and fair peer-review system, which is all easy to use. Visit http://www.dovepress.com/testimonials.php to read real quotes from published authors. 